\title{
Time-delayed coupled logistic capacity model in population dynamics
}

\author{
Manuel O. Cáceres* \\ Centro Atómico Bariloche, Instituto Balseiro and CONICET, 8400 Bariloche, Argentina \\ (Received 28 May 2014; revised manuscript received 14 July 2014; published 27 August 2014)
}

\begin{abstract}
This study proposes a delay-coupled system based on the logistic equation that models the interaction of a population with its varying environment. The integro-diferential equations of the model are presented in terms of a distributed time-delayed coupled logistic-capacity equation. The model eliminates the need for a prior knowledge of the maximum saturation environmental carrying capacity value. Therefore the dynamics toward the final attractor in a distributed time-delayed coupled logistic-capacity model is studied. Exact results are presented, and analytical conclusions have been done in terms of the two parameters of the model.
\end{abstract}

DOI: 10.1103/PhysRevE.90.022137

PACS number(s): $02.30 . \mathrm{Ks}, 87.23 . \mathrm{Cc}, 87.10 . \mathrm{Ed}$

\section{INTRODUCTION}

\section{A. Population growth}

The first law for population growth was given by Malthus in 1798 when he observed that population growth follows a geometrical progression. Under the conditions of unlimited sources, Malthus's law [1] can be expressed in the form $d N / d \tau=r N$, where $N(\tau)$ is the instantaneous population under consideration and $r$ is some proportionality constant. Later Verhulst in 1838 took into account the fact that the growth may stop due to the tendency of one organism to destroy others, or to the limit on the density of population which the environment can hold (i.e., limited food); then he postulated that the rate of the population growth was proportional to the product of the existing population and the difference between the total available resources and the resources used by the present population [2]. Denoting by $K$ the maximum population that a given amount of food can support, Verhulst's equation can be written in the form $d N / d \tau=r N(1-N / K)$. The solution of this equation gives the so-called Logistic curve, which has a characteristic $S$-like shape.

The continuing interest in the Logistic equation [3], and indeed some of its limitations, is reflected in many works which modify it for a wide variety of applications. Among then there are stochastic modifications considering random temporal variations into the environment $[4,5]$ and also taking into account pure deterministic modifications [6-9]. Many of these latter approaches attempt to consider the crucial point that in reality the carrying capacity $(K)$ cannot be considered as a constant. Positive changes in environment such as new resources elevate the carrying capacity, where a negative change, such as the advent of a toxic environment, will degrade the carrying capacity. Therefore it would be valuable to modify the Logistic equation so that the final asymptote depends on conditions while growth occurs. Such attempt have been recently presented coupling the evolution of the carrying capacity linearly with the population [10] and also in a nonlinear way [11].

Many ecosystems can be modified when external perturbations arise, and to emulate these facts most of the current models are achieved considering stochastic modifications in the dynamics $[4,5,12]$; these approaches lead to the study of

*caceres@cab.cnea.gov.ar population growth in terms of mean values and its fluctuations [13-15]. In the present work we will follow a quite different route to tackle this important ecological problem. We will modify Verhulst's dynamics by treating the carrying capacity as a state variable in the governing equation of the model. Thereby, any change to the environment will be reflected into the dynamics of the ecosystem. Another modification that we will introduce is the fact that a realistic environment does not change instantaneously to external perturbations [16], so we will take care of this fact by introducing a distributed time-delayed coupled Logistic-Capacity model.

\section{B. Pattern formation in population dynamics}

Another important chapter in the generalization of the Logistic model are the so-called reaction-diffusion equations [17]. In fact, population dynamics covers a wide spectrum of fields, and the formation of patterns in the evolution of bacterial colonies provides a good example of application [18]. In 1937, Fisher proposed a one-dimensional model: $\partial_{\tau} N(x, \tau)=$ $r N(1-N / K)+D \partial_{x}^{2} N$ for the spread of an advantageous gene in a population [19]. Since then nonlocal effects in the competition term have also been introduced to generalize Fisher's equation, in an attempt to improve the description of pattern formation in population dynamics [17,20,21] and also with the Allee effect [22]. For instance, in the case of bacteria, the diffusion of nutrients and/or the release of toxic substances can cause nonlocality in the interaction. Also nonlocal interactions with stochastic perturbations have recently been considered to find a characteristic time scale for the pattern formation [23]. In all these studies the nonlocality is introduced by considering the space variable in the description of the population growth.

In the present paper we will study a related but different approach, considering a zero-dimensional Logistic model with a carrying capacity coupled with distributed delays to the size of the population (nonlocality in time). Similar zerodimensional models have also been used to study oscillations in epidemic problems [24].

\section{The open-ended logistic-like growth}

Time-dependent forms for the carrying capacity, $K(\tau)$, have been successfully used to describe the enrichment of a lake by nutrients [25], to forecast product life cycles of electrical 
goods [26], to describe seasonal environments [27], and to show the changing micro-environment beneath and occlusion on healthy human skin [28]. For all these applications it is important that the carrying capacity is not treated as a constant. Thus it is valuable to modify the Logistic equation so that the asymptotic mass becomes a quantity which acclimates to growth conditions. This purpose is achieved by the open-ended Logistic-like growth model proposed by Thornley and France [10]. In that approach the carrying capacity $K(\tau)$ is coupled linearly to the size of the population (or mass) $N(\tau)$, and the coupling parameter is related to the ratio between the environmental development rate and the population growth rate. We have shown that Thornley and France's coupled Logistic-Capacity model can be rewritten as a coupled population growth model with distributed times delay, in fact using an exponential distribution of times [29].

In the present work we reinvestigate this problem by considering a more realistic distribution for the times delay. Then this generalization could allow for situations in which the carrying capacity encompasses the adjustment of the environment resulting from imposed changes (as occurs on the skin surface due to the application of an occlusion) [28]. So we will introduce a biparametric exponential distribution with two characteristic time scales: $\lambda^{-1}, t_{D}$. This distribution improves the description that relates the natural delay in the coupling between the population and the environment, which is more similar to many real situations (as, for example, occurs in the case of real diseases [24]). Our generalization allows a better description for the time-delay distribution, leading it to a broad or narrow coupling, between the carrying capacity and the population, depending on the values of parameters $\lambda^{-1}$ and $t_{D}$. When $t_{D} \sim 0$, the parameter $\lambda^{-1}$ is the coupling parameter as in the Logistic-Capacity model [10], but in general the parameter $t_{D}$ will characterize a time scale for a sharp distribution of times delay. By taking $t_{D}=0$ we recover Thornley and France's model. The time scale $t_{D}$ also permits mass at the inflexion point to be a higher fraction than in Verhulst's model or in Thornley and France's model. The time scale $t_{D}$ also gives the possibility to modify the value of the attractor $N(\tau=\infty) \equiv \bar{N}$ at the stationary state. In addition we will show that the presence of $t_{D} \neq 0$ can introduce oscillations and instabilities in the system, a situation which is of interest in problems involving diseases (in the case $t_{D} \rightarrow 0$ the system is always stable and does not present any oscillations).

\section{THE COUPLED LOGISTIC-CAPACITY MODEL}

In a recent work [29] it has been proved that the open-ended Logistic-base model, introduced by Thornley and France [10],

$$
\begin{aligned}
d \tilde{N} / d \tilde{t} & =a \tilde{N}(1-\tilde{N} / \tilde{K}), \\
d \tilde{K} / d \tilde{t} & =b(\tilde{N}-\tilde{K}),
\end{aligned}
$$

is equivalent to the exponential distributed time-delay Logistic model:

$$
\begin{aligned}
d N / d t & =N(1-N / K), \\
K(t) & =\int_{0}^{\infty} \lambda e^{-\lambda s} N(t-s) d s .
\end{aligned}
$$

Here I have rescaled Eqs. (1) and (2) using the substitutions $N \rightarrow b \tilde{N}, K \rightarrow b \tilde{K}, t \rightarrow a \tilde{t}$, where $\lambda=b / a$ is the ratio between the environmental development rate and the population growth rate.

Note that to have a one-to-one correspondence with the original Thornley and France's model, we must relate the initial condition $K(0)$ (in rescaled units) to the prefunction $\varphi(s)$ associated with Eq. (4) [i.e., $N(-s), s \in(\infty, 0)$ ] in the form

$$
K(0)=\int_{0}^{\infty} \lambda e^{-\lambda s} N(-s) d s=\int_{-\infty}^{0} \lambda e^{\lambda s} \varphi(s) d s .
$$

Therefore in general $K(0)$ is proportional to the Laplace transform of the prefunction. Thus, if we take as a prefunction a constant $\varphi(s)=\varphi$, we get $K(0)=\varphi$.

\section{Generalizing Thornley and France's model}

Following reference [29] it is possible to go one step forward and to generalize the exponential distributed time-delay model [Eq. (4)] by considering a general delay distribution:

$$
\begin{aligned}
K(t) & =\int_{0}^{\infty} G(s) N(t-s) d s=\int_{-\infty}^{t} G(t-x) N(x) d x \\
1 & =\int_{0}^{\infty} G(s) d s .
\end{aligned}
$$

Here a probability distribution $G(s)$ has been introduced to take into account a more realistic delay dynamics in the coupled Logistic-Capacity model.

The general condition to study the stability at the attractor $N=K=\bar{N}$ can be written in the form

$$
c+i \omega=1-\int_{0}^{\infty} G(s) e^{(c+i \omega) s} d s
$$

here I have used a perturbation $n(t) \propto \exp (-c t+i \omega t)$ to do the linear stability analysis around the attractor $\bar{N} \neq 0$; see Appendix A in Ref. [29].

Using the exponential distribution $G(s)=\lambda e^{-\lambda s}$ in Eq. (8) we obtain $c+i \omega=1+\lambda /(c+i \omega-\lambda)$, indicating that the attractor is stable and there are no oscillations in Thornley and France's model.

To generalize the exponential distributed time delay, we propose here to use the Laplace distribution. This probability distribution is a biparametric function with a sharp peak at $s=t_{D}$ :

$G(s)=\mathcal{N} \lambda \exp \left(-\lambda\left|s-t_{D}\right|\right), \quad \lambda>0, t_{D} \geqslant 0, s \in(0, \infty)$,

where $\mathcal{N}=\mathcal{N}\left(\lambda, t_{D}\right)$ is the normalization constant (see Appendix A). Using this distribution in Eq. (6) allows us to have a much more interesting coupling between the population and the carrying capacity. This fact ultimately will lead to an inertial differential equation for the carrying capacity $K$, which will have a nonlinear term [coming from $\dot{N}(t)$ ], and a linear delay coupling with the population, i.e., $N\left(t-t_{D}\right)$. In addition, this generalization allows us to recover Thornley and France's model by taking $t_{D}=0$. 


\section{THE TIME-DELAYED COUPLED LOGISTIC CARRYING CAPACITY MODEL}

In what follows we will concentrate on the analysis of the delay-coupled Logistic-Capacity model:

$$
\begin{aligned}
\dot{N} & =N(1-N / K), \\
K(t) & =\int_{0}^{\infty} G(s) N(t-s) d s,
\end{aligned}
$$

when time delay is characterized by the Laplace distribution [Eq. (9)]. In this case the stability condition Eq. (8) reads

$$
(z-1)(z-\lambda)=\frac{(z-\lambda) G(0)}{z+\lambda}+\frac{2 \lambda G\left(t_{D}\right) e^{z t_{D}}}{(z+\lambda)},
$$

where $z \equiv(c+i \omega)$ and $G(0), G\left(t_{D}\right)$ follow from the definition of $G(s)$; see Eqs. (A9) and (A10) and Appendix B.

\section{A. The case $t_{D}=0$}

In the particular case when $t_{D}=0$ we re-obtain the previous stability analysis:

$$
c+i \omega=1+\frac{\lambda}{(c+i \omega-\lambda)}, \quad t_{D}=0 .
$$

This equation can be transformed into a quadratic one, getting (apart from the trivial solution $z=0$ ) the exact solution:

$$
\left.\begin{array}{rl}
c & =1+\lambda \\
\omega & =0
\end{array}\right\}, \quad t_{D}=0 .
$$

This solution indicates that the attractor $\bar{N}$ is stable and there are no oscillations for any value of $\lambda$ if $t_{D}=0$. The value of the attractor will depend on the initial values $N(0), K(0)$ needed to solve Thornley and France's dynamics:

$$
\begin{aligned}
& \dot{N}=N(1-N / K), \\
& \dot{K}=\lambda(N-K) .
\end{aligned}
$$

\section{On the attractor $\bar{N}$ for Thornley and France's model}

This model has infinitely many stationary solutions along the line $N=K$. Therefore initial conditions $N(0)=K(0)$ will have no dynamical behavior. Within this model the choice of initial condition determines the asymptotic carrying capacity. In addition the system requires the initial value of the carrying capacity to be higher value than the initial population in order to observe the population growth. If the initial carrying capacity is lower than the initial population, the population decreases toward the carrying capacity $[10,11]$.

In order to find an expression for $\bar{N}$ we proceed in the following way. First, from Eq. (14) we take the second derivative of $K(t)$, then we write the equivalent set of dynamics equations [i.e., a three-dimensional system]:

$$
\begin{aligned}
\dot{N} & =N(1-N / K), \\
\dot{K} & =V, \\
\dot{V} & =\lambda N(1-N / K)-\lambda V .
\end{aligned}
$$

Note that these equations should be worked out with the set of initial conditions $N(0), K(0), V(0)$, where we must use

$$
V(0)=\lambda[N(0)-K(0)]
$$

in order to have a one-to-one equivalence with Eqs. (13) and (14). The stationary state associated with this threedimensional system is characterized by $N(\infty)=K(\infty) \rightarrow \bar{N}$ and $V(\infty) \rightarrow 0$. Using these facts we can formally integrate Eq. (17) to get

$$
\begin{aligned}
\int_{0}^{t} \dot{V}(s) d s & =\lambda \int_{0}^{t} \dot{N}(s) d s-\lambda \int_{0}^{t} V(s) d s \\
& =\lambda[N(t)-N(0)]-\lambda^{2} \int_{0}^{t}[N(s)-K(s)] d s ;
\end{aligned}
$$

then, taking $t \rightarrow \infty$ we obtain

$$
K(0)-\bar{N}=\lambda \int_{0}^{\infty}[K(s)-N(s)] d s .
$$

This formula is an Areas's rule from which the attractor $\bar{N}=N(\infty)$ can be interpreted. It is simple to check this rule with the numerical solutions presented in Refs. [10,11]. Interestingly a similar Areas's rule can also be obtained for the general case $t_{D} \neq 0$; this will be presented in the next section (see Appendix C).

\section{B. The case $\lambda t_{D} \ll 1$}

In the particular situation when $\lambda t_{D} \ll 1$ and if we try to introduce a perturbation analysis, we soon run into trouble. For example from Eq. (10) the stability condition would read

$$
\begin{aligned}
(z-\lambda)(z-1)= & \lambda+\left[\left(\lambda t_{D}\right)^{2}-\frac{4}{3}\left(\lambda t_{D}\right)^{3}+\frac{23}{12}\left(\lambda t_{D}\right)^{4}+\cdots\right] z \\
& +\left[\frac{1}{3}\left(\lambda t_{D}\right)^{2}-\frac{5}{12}\left(\lambda t_{D}\right)^{3}+\cdots\right] t_{D} z^{2} \\
& +\left[\frac{1}{12}\left(\lambda t_{D}\right)^{2}+\cdots\right] t_{D}^{2} z^{3}+\cdots
\end{aligned}
$$

We see that to $O\left[\left(\lambda t_{D}\right)^{2}\right]$ there are contributions of all order in $t_{D}^{n} z^{n+1}$ indicating the complex structure of its analysis. So to study the stability analysis we will proceed in a different way as we shall present in the next section.

\section{The case $t_{D} \neq 0$}

To characterize the stability, in the general case, we have to work out Eq. (10); but this is a transcendental equation which must be solved numerically. Nevertheless, some important information can be analytically obtained.

\section{Stability analysis without oscillations (case $\omega=0$ )}

Consider the situation when there are not oscillations, in this case $z=c$ and we can write from Eq. (10)

$$
\begin{aligned}
(c-1)(c-\lambda) & =\frac{(c-\lambda) G(0)}{c+\lambda}+\frac{2 \lambda G\left(t_{D}\right) e^{c t_{D}}}{(c+\lambda)} \\
& =\frac{G\left(t_{D}\right) e^{c t_{D}}}{(c+\lambda)}\left(2 \lambda+(c-\lambda) e^{-(\lambda+c) t_{D}}\right) \\
& \equiv f\left(\lambda, t_{D}, c\right) .
\end{aligned}
$$


Here the function $f\left(\lambda, t_{D}, c\right)$ is an exponential-like function which can easily be plotted. In particular we get the following important values:

$$
f\left(\lambda, t_{D}, c\right) \Rightarrow\left\{\begin{array}{l}
f(\lambda, 0, c)=\lambda \\
f\left(0, t_{D}, c\right)=0 \\
f\left(\lambda, t_{D},-\infty\right)=\lambda /\left(2 e^{\lambda t_{D}}-1\right) \\
f\left(\lambda, t_{D}, \infty\right) \rightarrow \infty \\
f\left(\lambda, t_{D}, 0\right)=\lambda .
\end{array}\right.
$$

Therefore, we can plot the parabola $(c-1)(c-\lambda)$ against $f\left(\lambda, t_{D}, c\right)$ as a function of $c$, and from the intersection points we get the roots showing the stability of the system if $c>0$. If there were negative roots, $c<0$, this would indicate an instability (the marginal situation $c=0$ will be presented in the next section). In particular for $t_{D}=0$ we recover the results of the previous section [Eq. (12)].

From the behavior of the function $f\left(\lambda, t_{D}, c\right)$ it is simple to realize that the roots are only positive. Nevertheless, for some values of the parameters $\lambda, t_{D}$ (in particular if $\lambda t_{D} \gg 1$ ) there are no intersection points; this fact signals the transition to a different scenario where there could be some instable oscillatory behavior. In Figs. 1(a) and 1(b) we present this analysis for two cases $\lambda \lessgtr 1$ and several values of $t_{D}$.

\section{Andronov-Landau-Hopf bifurcation (case $c=0$ )}

When $z$ is pure imaginary, i.e., $z=i \omega$, it is known that there may be an Andronov-Landau-Hopf bifurcation in the system, corresponding to a pair of complex conjugated eigenvalues crossing the imaginary axis, separating the left- and right-hand complex plane. Substituting $z=i \omega$ in Eq. (10) we obtain

$$
\begin{aligned}
& -(i \omega-1)\left(\omega^{2}+\lambda^{2}\right) \\
& \quad=(i \omega-\lambda) G(0)+2 \lambda G\left(t_{D}\right) e^{i \omega t_{D}}, \quad t_{D} \geqslant 0 .
\end{aligned}
$$

Separating real and imaginary parts, and after some algebra, we obtain

$\frac{1}{2}\left(1-\frac{1}{\lambda}\right) \frac{G(0)}{G\left(t_{D}\right)} \omega t_{D}=\omega t_{D} \cos \omega t_{D}+t_{D} \sin \omega t_{D}, \quad t_{D}>0$.

We are interested in the intersection points $\omega t_{D}$ from the plot of the left- and right-hand sides of Eq. (25). Depending on the values of $\lambda$ and $t_{D}$ there may or may not be roots $\omega t_{D}$ of this equation indicating a bifurcation. Thus, we will plot the zero cross points of the function

$$
\omega t_{D} \cos \omega t_{D}+t_{D} \sin \omega t_{D}-g\left(\lambda, t_{D}\right) \omega t_{D},
$$

where

$$
g\left(\lambda, t_{D}\right)=\frac{1}{2}\left(1-\frac{1}{\lambda}\right) e^{-\lambda t_{D}} .
$$

In Fig. 2(a) the zero cross points can be seen in the interval $\omega t_{D} \in(0,2 \pi)$ for fixed $t_{D}$ and several values of $\lambda$. For $\lambda=1$, and for large values of $\lambda \gg 1$ the zero cross points coincide with the zeros of the function $\omega t_{D} \cos \omega t_{D}+t_{D} \sin \omega t_{D}$ (we remind readers that $t_{D}$ and $\lambda$ are dimensionless). For $\lambda<1$ there is a minimum value $\lambda_{\min }=\lambda_{\min }\left(t_{D}\right)$ after which there are not any more roots, so the only solution of Eq. (25) is the trivial one. In Fig. 2(b) the zero cross points are shown for fixed $\lambda$ and several values of $t_{D}$. For large values of $t_{D} \gg 1$
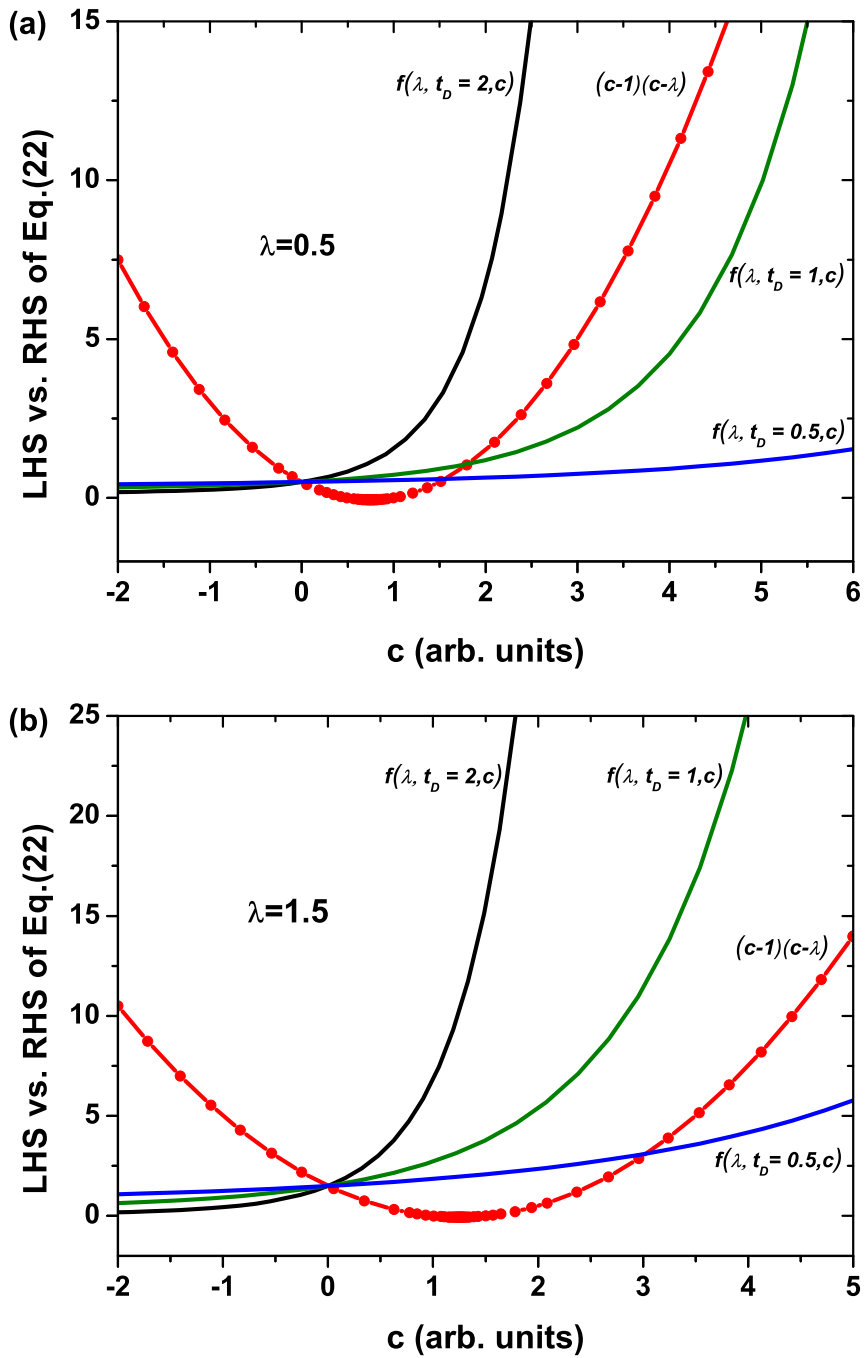

FIG. 1. (Color online) Stability analysis for $\lambda \lessgtr 1$ and for several values of $t_{D}$ using Eq. (22) to show graphically possible intersection points [roots of Eq. (10) using $z=c$, i.e., with $\omega=0$ ].

the roots are the same as the one from the function $\sin \omega t_{D}$ independently of the value of $\lambda$. For small values of $t_{D} \ll 1$ the roots are the same as the roots of

$$
\begin{aligned}
X \cos X & \simeq \frac{1}{2}\left(1-\frac{1}{\lambda}\right) X, \\
X & =\omega t_{D} .
\end{aligned}
$$

\section{The general case $(c \neq 0, \omega \neq 0)$}

When $z=c+i \omega$, from Eq. (10) we arrive to

$$
\begin{aligned}
& (c+i \omega-1)\left[(c+i \omega)^{2}-\lambda^{2}\right] \\
& \quad=(c+i \omega-\lambda) G(0)+2 \lambda G\left(t_{D}\right) e^{(c+i \omega) t_{D} .} .
\end{aligned}
$$

The real part of Eq. (26) is

$$
\begin{aligned}
& (c-1)(c-\lambda)+\frac{\omega^{2}(1-3 c)}{(c+\lambda)} \\
& =\frac{G\left(t_{D}\right) e^{c t_{D}}}{(c+\lambda)}\left[2 \lambda \cos \omega t_{D}+(c-\lambda) e^{-(\lambda+c) t_{D}}\right] .
\end{aligned}
$$



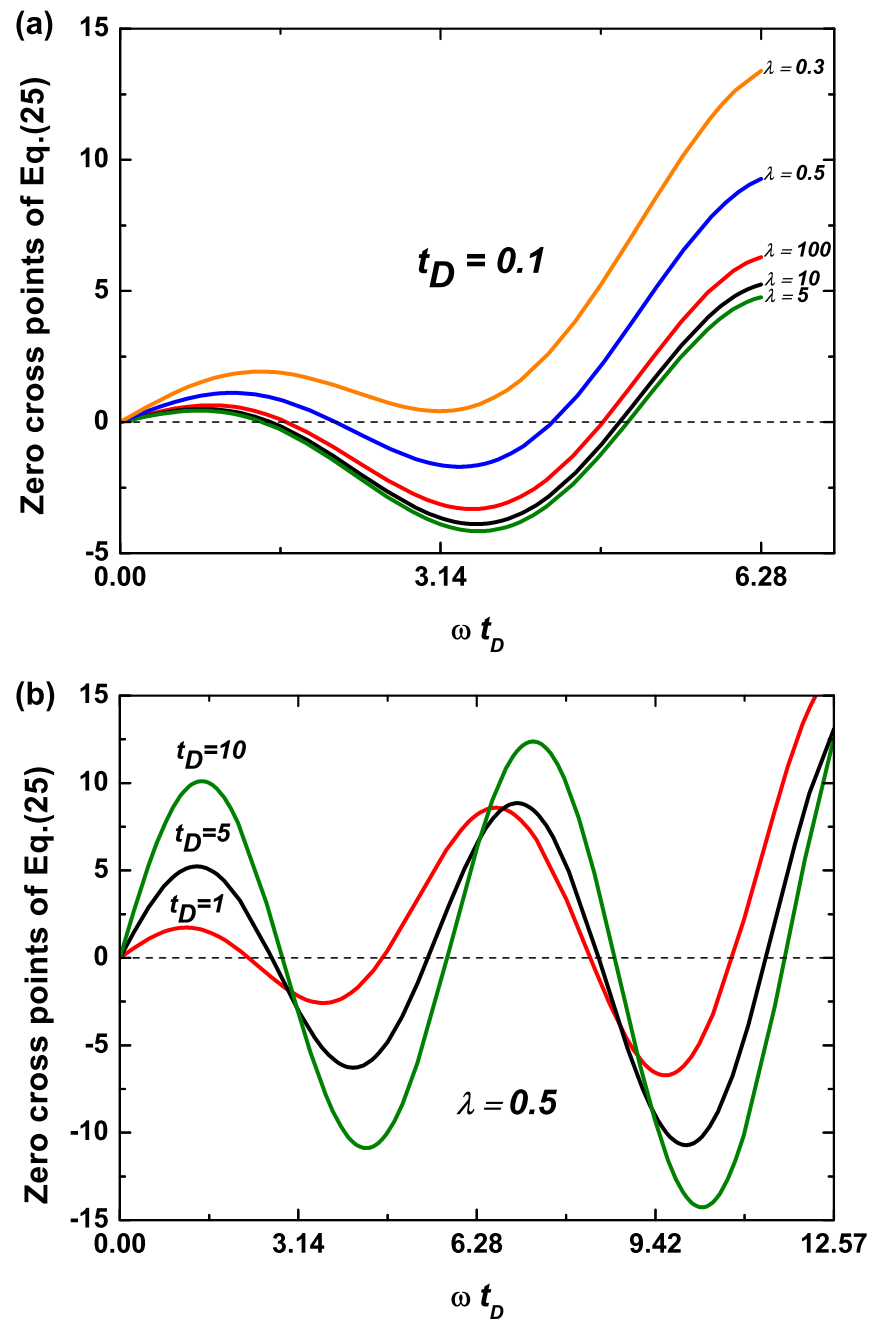

FIG. 2. (Color online) Andronov-Landau-Hopf bifurcation analysis from Eq. (10) using $z=i \omega$ (with $c=0$ ) for several values of $\lambda$ and $t_{D}$. (a) Zero cross points from Eq. (25) in the interval $\omega t_{D} \in(0,2 \pi)$ for $t_{D}=10^{-1}$ and $\lambda(=0.3,0.5,5,10,100)$. (b) Zero cross points from Eq. (25) in the interval $\omega t_{D} \in(0,4 \pi)$ for $\lambda=0.5$ and $t_{D}(=1,5,10)$.

Taking the imaginary part of Eq. (26) we get

$\omega\left(3 c^{2}-2 c\right)-\omega\left(\omega^{2}+\lambda^{2}\right)=\omega G(0)+2 \lambda G\left(t_{D}\right) e^{c t_{D}} \sin \omega t_{D}$.

Using Eq. (27) with $\omega=0$ we obtain Eq. (22). Making the combination $\frac{1}{\omega}$ Eq. (28) plus $\lambda$ Eq. (27) and taking $c=0$, we obtain Eq. (25). To solve the general situation, when $c \neq 0$ and $\omega \neq 0$, we must work out numerically Eqs. (27) and (28).

\section{The limit case $\lambda \rightarrow \infty$ with $t_{D} \neq 0$}

In this extreme situation it follows that $G(s) \rightarrow \frac{\lambda}{2}$ $\exp \left(-\lambda\left|s-t_{D}\right|\right) \rightarrow \delta\left(s-t_{D}\right)$; therefore

$$
K(t)=\int_{0}^{\infty} G(s) N(t-s) d s=N\left(t-t_{D}\right) .
$$

The stability condition follows from Eq. (10) taking with care the limit $\lambda \rightarrow \infty$; therefore we get the transcendental equation:

$$
\begin{aligned}
& z=1-e^{z t_{D}}, \\
& z=c+i \omega .
\end{aligned}
$$

This equation shows that the system is at the boundary of stability if $t_{D}<1$; see also Appendix D.

\section{EXACT DIFFERENTIAL EQUATIONS FOR THE DELAY-COUPLED LOGISTIC MODEL}

The delay-coupled Logistic-Capacity model [using the Laplace distribution $G(s)$ ]

$$
\begin{aligned}
\dot{N} & =N(1-N / K), \\
K(t) & =\int_{0}^{\infty} G(s) N(t-s) d s,
\end{aligned}
$$

where $G(t)$ fulfills the two-sided Green evolutions (see Appendix A)

$$
\begin{array}{ll}
d G^{+} / d t=+\lambda G^{+}(t), & t<t_{D} \\
d G^{-} / d t=-\lambda G^{-}(t), & t>t_{D}
\end{array}
$$

can be written as a set of differential equations for a set of state variables (see Appendix B in Ref. [29]). In general, to be able to find a close differential equation for the carrying capacity $K(t)$ it is necessary that the probability density $G(s)$ fulfills a linear evolution equation, otherwise it is not possible to close the system.

Taking two times the time derivative of $K(t)$, from Eq. (31) it is possible to arrive at an exact set of equations for the present delay-coupled model:

$$
\begin{aligned}
\dot{N}= & N(1-N / K), \\
\dot{K}= & V, \\
\dot{V}= & G(0) N(1-N / K)-2 \lambda G\left(t_{D}\right) N\left(t-t_{D}\right) \\
& +\lambda G(0) N+\lambda^{2} K .
\end{aligned}
$$

In the case $t_{D}=0$ this set of equations reduces to the system of equations given in Eqs. (15)-(17). We remind that in the case $t_{D}=0$ we recover the same dynamics as in the Thornley and France model; i.e., Eqs. (13) and (14) are equivalents to Eqs. (3) and (4). Note that if $t_{D} \neq 0$ the coupling has inertia and is now written in terms of $\ddot{K}$, i.e., a second order differential equation. The structure of this equation is nonlinear and with a delayed term proportional to the population at previous time $N\left(t-t_{D}\right)$. This delay structure is induced by the sharp peak around $t_{D}$ in the Laplace distribution.

Equation (36) can be used to find an approximated evolution for $V(t)$ if we introduce a perturbation in the characteristic time $t_{D}$. For example using a Taylor expansion: $N\left(t-t_{D}\right)=$ $\left[N(t)-\dot{N}(t) t_{D}+\ddot{N}(t) t_{D}^{2} / 2+\cdots\right]$, we can find [to any order 
$O\left(t_{D}^{n}\right)$ ] a set of coupled equations for the system which will be valid for a short elapse of time; see the next section.

As before the set of initial conditions to be used are $N(0), K(0), V(0)$, but in this case $V(t)$ has a much more complex structure than in the case $t_{D}=0$. So in order to write an expression for $V(0)$ [in terms of the parameters $N(0)$ and $K(0)]$ we now use the definition of $V(t)=\dot{K}(t)$ :

$$
\begin{aligned}
V(t)= & \frac{d}{d t} \int_{-\infty}^{t} G(t-s) N(s) d s \\
= & G(0) N(t)-\lambda \int_{-\infty}^{t-t_{D}} G^{+}(t-s) N(s) d s \\
& +\lambda \int_{t-t_{D}}^{t} G^{-}(t-s) N(s) d s .
\end{aligned}
$$

Putting $t=0$ in Eq. (37) and using a constant prefunction $N(-s)=\varphi[$ as we commented in Eq. (5)] we get

$$
V(0)=G(0)[N(0)-\varphi] .
$$

From this equation we see that the constant $\varphi$ must be equal to $K(0)$ in order to recover the same initial value problem of Eqs. (13) and (14) in the limit $t_{D} \rightarrow 0$.

\section{Areas's rule for $\bar{N}$ in the case $t_{D} \neq 0$}

In the same way as we did with the evaluation of the stationary state associated with the set of Eqs. (15)-(17), we now proceed to make equivalent calculations in the case $t_{D} \neq 0$. The stationary state for the system of Eqs. (34)-(36) is as before characterized by $N(\infty)=K(\infty) \rightarrow \bar{N}$ and $V(\infty) \rightarrow$ 0 , but now $V(t)$ is given by Eq. (37); note that in the case $t_{D}=0$ we recover the expression $V(t)=\lambda(N(t)-K(t))$.

After some algebra (see Appendix C) we arrive at

$$
\begin{aligned}
G(0)[K(0)-\bar{N}]= & \lambda^{2} \int_{0}^{\infty}[K(s)-N(s)] d s \\
& -2 \lambda G\left(t_{D}\right) t_{D} K(0) .
\end{aligned}
$$

Equation (39) is an exact result which gives an Areas's rule for $\bar{N}$ in the case $t_{D} \neq 0$; this formula reduces to Eq. (20) in the limit $t_{D} \rightarrow 0$. Comparing this Areas's rule with the one for Thornley and France's model [Eq. (20)], we see that apart from the constant $\lambda^{2} / G(0)$ (that depends on $t_{D}$ ) the difference is given by the positive quantity $2 \lambda G\left(t_{D}\right) K(0) t_{D}$. Therefore, in the case when $[K(0)-N(0)]>0$ the presence of a delay time $t_{D} \neq 0$ reduces the gap difference $\Delta \equiv[K(0)-\bar{N}]$ by the quantity $2 \lambda G\left(t_{D}\right) t_{D} K(0) / G(0)$.

Thus, for many organisms, where the final mass depends on conditions during growth, application of the present generalized coupled-Logistic model is feasible. For example, in the case of a small time delay, $t_{D} \simeq 0$, the gap difference is given by

$$
\begin{aligned}
{[K(0)-\bar{N}]=} & \lambda\left(1+2 \lambda t_{D}\right) \int_{0}^{\infty}[K(s)-N(s)] d s \\
& -2 \lambda t_{D} K(0)+O\left(t_{D}^{2}\right) .
\end{aligned}
$$

\section{SOLUTIONS FOR THE GENERALIZED COUPLED LOGISTIC MODEL UP TO $O\left(t_{D}\right)$}

In this section we are going to work out Eqs. (34)-(36) up to $O\left(t_{D}\right)$. Thus we can write

$$
\begin{aligned}
\dot{N}= & N(1-N / K) \\
\dot{K}= & V \\
\dot{V}= & G(0) N(1-N / K)-2 \lambda G\left(t_{D}\right)\left[N-\dot{N} t_{D}+O\left(t_{D}^{2}\right)\right] \\
& +\lambda G(0) N+\lambda^{2} K \\
= & {\left[G(0)+2 \lambda G\left(t_{D}\right) t_{D}\right] N(1-N / K) } \\
& +\left[\lambda G(0)-2 \lambda G\left(t_{D}\right)\right] N+\lambda^{2} K+O\left(t_{D}^{2}\right) \\
= & \lambda N(1-N / K)+\lambda^{2}(K-N)+O\left(t_{D}^{2}\right) .
\end{aligned}
$$

The first important point to be noted from Eq. (43) is that to order $O\left(t_{D}\right)$ there are not corrections in the dynamics evolution, for the only difference appears in the initial condition for $V(0)$; see Eq. (38).

We have solved numerically these equations for two sets of initial conditions $\{N(0), K(0)\}$ and using the prescription Eq. (38) for assigning a value to $V(0)$, which to order $O\left(t_{D}\right)$ can be written as $V(0) \simeq \lambda\left(1-2 \lambda t_{D}\right)[N(0)-K(0)]$. In Fig. 3 we show these solutions for two values of $\lambda$ and different values of the parameter $t_{D}$ inside the domain of stability. A reasonable criterion to establish how good is the approximation used in Eq. (43) is to look at the time $t^{\prime}$ when the function $V(t)$ leaves its asymptotic zero value [we know that in the stationary state $V(\infty)=0$ ]. It is easy to see from Fig. 3 that when $V(t)$ reachs the zero value the functions $N(t)$ and $K(t)$ are in a plateau, indicating that they have arrived at the stationary state $\bar{N}$. On the other hand the value of $\bar{N}$ is in accordance with the Areas's rule [Eq. (39)].

TABLE I. Values for the differences $\Delta \equiv[K(0)-\bar{N}]$ calculated from Eq. (39) using a numerical integration of the solutions of Eqs. (41)(43). In the case $\lambda=0.5$ two different initial conditions $\{N(0), K(0)\}$ were used. In the case $\lambda=0.1$ and $\lambda=0.05$ we use only $N(0)=0.1$, $K(0)=0.2$. The predicted values of $\Delta$ show very good agreement with the plateau observed in Fig. 3 . The last column corresponds to the Thornley and France model (the exponential distributed delay case, $t_{D}=0$ ).

\begin{tabular}{lccc}
\hline \hline & $t_{D}=10^{-2}$ & $t_{D}=10^{-3}$ & $t_{D}=0$ \\
\hline$\lambda=0.1$ & $\Delta=0.011821$ & $\Delta=0.0121746$ & $\Delta=0.0122121$ \\
$\lambda=0.5$ & $\{N(0)=0.1, K(0)=0.4\}$ & $\Delta=0.147724$ & $\Delta=0.147976$ \\
$\lambda=0.5$ & $\{N(0)=0.1, K(0)=0.2\}$ & $\Delta=0.0410764$ & $\Delta=0.0412352$ \\
$\lambda=0.05$ & $t_{D}=0.5$ & $t_{D}=10^{-4}$ & $t_{D}=0$ \\
\hline \hline
\end{tabular}



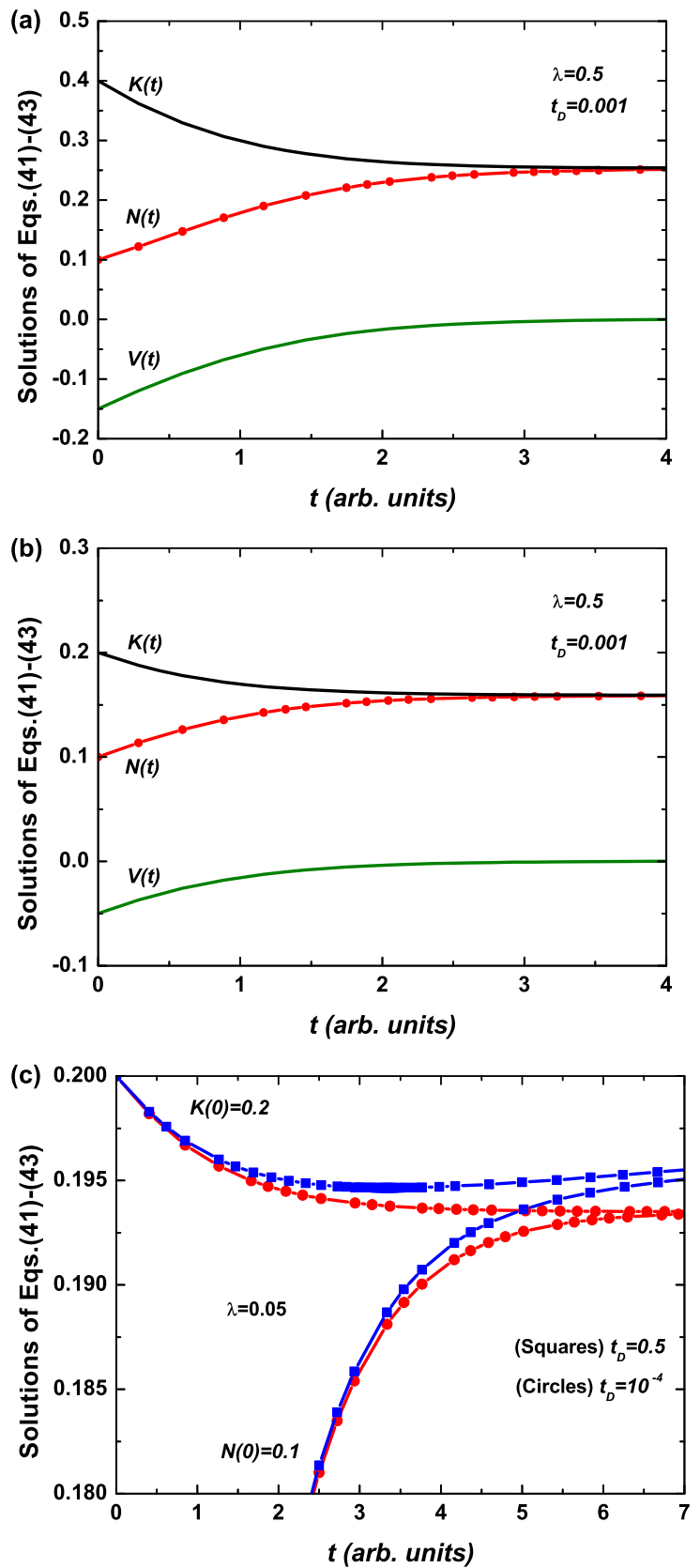

FIG. 3. (Color online) Numerical solutions from Eqs. (41)-(43) as a function of time for two set of initial conditions $\{N(0), K(0)\}$, using the Laplace distributed time-delayed coupled Logistic-Capacity model. Two values of $\lambda(=0.05,0.5)$ and different values of $t_{D}$ have been used. Solutions correspond to the approximation $O\left(t_{D}\right)$, so these evolutions are valid only for a short elapse of time. This crucial time $t^{\prime}$ can be observed by looking at the behavior of $V(t)$. This time $t^{\prime}$ corresponds to the time when having arrived the function at its stationary state, $V(\infty)=0$, it starts to abandon its plateau; this means that after this elapse of time the approximation $O\left(t_{D}\right)$ used in Eq. (43) does not work any more. The asymptotic value $\bar{N}$ can be observed to be in agreement with the Areas's rule Eq. (39) before the approximation $O\left(t_{D}\right)$ starts to fail. (a) The temporal behavior of $N(t), K(t)$, and $V(t) \equiv \dot{K}(t)$ for $N(0)=0.1$ and $K(0)=0.4$, using $\lambda=0.5$ and $t_{D}=10^{-3}$. (b) $N(t), K(t), V(t)$ for $N(0)=0.1$ and $K(0)=0.2$, for the same values of $\lambda, t_{D}$. (c) Corresponds to $\lambda=0.05$ with initial conditions $N(0)=0.1$ and $K(0)=0.2$, for two values of the delay time: $t_{D}=10^{-4}$ (circles) and $t_{D}=0.5$ (squares).

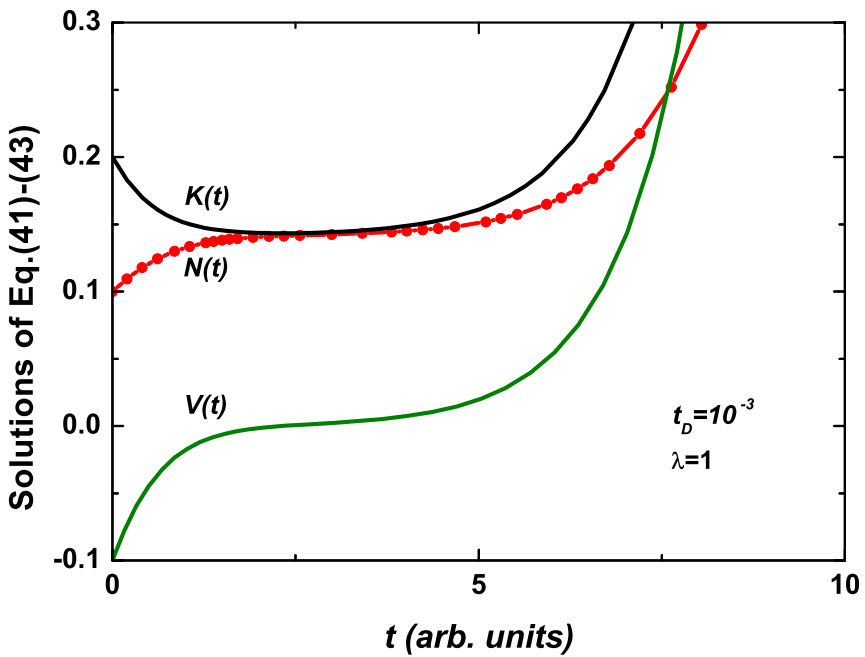

FIG. 4. (Color online) Numerical solutions of $N(t), K(t), V(t)$ from Eqs. (41)-(43) using $\lambda=1, t_{D}=10^{-3}$. Here it is possible to see that $V(t)$ crosses the zero value without approaching any plateau-like behavior as its does in Fig. 3 .

In Table I we present several values for the difference $\Delta \equiv[K(0)-\bar{N}]$ as a function of the parameters $\lambda, t_{D}$. This table shows a good agreement between the Areas's rule and the plateau reached by $N(t), K(t)$ from the numerical solutions, $N(t), K(t)$, of Eqs. (41)-(43). In addition the stability criterion given in Eqs. (27) and (28) has also been checked.

In Fig. 4 we show the time evolution of $N(t), K(t), V(t)$ in the neighborhood of the instability using the values $\lambda=1$, $t_{D}=0.001$.

Higher order analysis of the solutions can be studied considering the next contribution in the Taylor expansion of $N\left(t-t_{D}\right)$. Also a nonperturbative analysis from the integrodifferential Eqs. (30) and (31) could be done in order to compare the solution $N(t)$ for any $\left\{\lambda, t_{D}\right\}$ with the numerical evaluation of the exact stability criterion given in Eqs. (27) and (28); these and other numerical results will be reported elsewhere.

\section{DISCUSSION}

We have presented a Logistic-like model in terms of coupled equations considering $N$ and $K$ as state variables. The important point in our approach is that the carrying capacity, $K$, is coupled linearly to the population size, $N$, but introducing a multiple time delay coupling, which in this case is represented by a biparametric exponential probability distribution $G(s)$ (see Appendix A). Then we have shown that the integrodiferential system of Eqs. (30) and (31) is equivalent to the set of differential Eqs. (34)-(36). Interestingly the system of Eqs. (34) and (36) has inertia and a delay (linear) term proportional to the population in a previous time $N\left(t-t_{D}\right)$. We have numerically solved the set of differential Eqs. (41)-(43), which correspond to a first order approximation in the Taylor expansion of $N\left(t-t_{D}\right)$, showing good agreement with the theoretical predictions from the stability analysis Eqs. (27) and (28), as well as with the Areas's rule. In fact, our Areas's rule [Eq. (39)] provides a useful way to interpret the value of 
the attractor for distributed time-delayed Logistic-like models. We have proved that in a distributed time-delayed coupled dynamics the difference $[K(0)-\bar{N}]$ (i.e., initial value of the carrying capacity minus the final value of the attractor) is modified by the quantity $2 \lambda G\left(t_{D}\right) t_{D} K(0) / G(0)$; see Eq. (40) for a small approximation to $O\left(t_{D}\right)$.

Many biological applications require a late inflexion point toward a higher fraction than the one-half of the final asymptotic mass as provided by the Verhulst model [10]. Our model shows this functionality moving the inflexion point to higher values of the final mass. In addition the present approach gives a rich oscillatory instability behavior when $t_{D} \neq 0$.

The present approach also includes the possibility to recover the previously reported coupled Logistic-Capacity model [10] by taking the time scale $t_{D}$ going to zero. In particular we have investigated the cause of instabilities by changing the parameters $\lambda, t_{D}$ of the model. These results are of importance because they could often signal changes in the dynamics of complex ecosystems. Our approach can be considered as a straightforward extension of the Thornley and France model, but showing, however, a very different behavior. A striking feature is the appearance of instabilities and oscillatory behaviors when $t_{D} \neq 0$, which are important facts to be taken into account in problems involving diseases.

Realistic models in ecosystems must account for the many factors producing changes in the environment; this is an extremely difficult issue which is currently under active investigation. Nevertheless, by incorporating a distributed time delay in the coupling between the carrying capacity and the population size we have been able to generalize the Logistic equation in a satisfactory and treatable way, which promotes the idea of the interrelation that coexists between the environment and the population. In general the integro-differential system characterized by Eqs. (3) and (6) is a valid model for any $G(s)$; then its stability analysis can be studied by using Eq. (8), for example, using a Gamma probability distribution as was used to study problems involving diseases [24]. The only difficulty lies in that if $G(s)$ does not have a Green's function evolution, it is not possible to find an equivalent set of differential equations for the system as we did in the present work.

Future extensions of this work will include a further study of the instabilities and the explicit incorporation of a noise term in order to study the characteristic time scale of the pattern formation. This future work will be done by using the first passage time techniques [29].

\section{ACKNOWLEDGMENT}

M.O.C. acknowledges grants from: SECTyP, No. 06/C353, Universidad Nacional de Cuyo, Argentina; and from CONICET PIP 90100290, Argentina.

\section{APPENDIX A: ON THE LAPLACE PROBABILITY DISTRIBUTION}

To generalize the exponential time delay occurring in Eq. (4) we propose here to use the Laplace distribution. This probability is a biparametric function with a sharp peak at

$$
\begin{aligned}
& s=t_{D}: \\
& G(s)=\mathcal{N} \lambda \exp \left(-\lambda\left|s-t_{D}\right|\right), \quad \lambda>0, t_{D} \geqslant 0, s \in(0, \infty),
\end{aligned}
$$

where the constant $\mathcal{N}$ is given by a normalization condition in the positive domain, i.e., $\int_{0}^{\infty} G(s) d s=1$; then

$$
\mathcal{N}=\left(2-e^{-\lambda t_{D}}\right)^{-1} \text {. }
$$

When $\lambda \gg 1$ Laplace's probability distribution is a narrow function around $t_{D}$, and in the opposite case when $\lambda \ll 1$ the distribution is a broad function with an exponential decay in the long-time regime $s \gg t_{D}$. In the case $t_{D}=0$ we recover the exponential distribution $G(s)=\lambda e^{-\lambda s}$. For $t_{D} \neq 0$ the limit $\lambda \rightarrow \infty$ corresponds to the fixed delay case $G(s) \rightarrow \delta\left(s-t_{D}\right)$.

Note that the Laplace distribution fulfills a two-sided Green function evolution:

$$
\begin{aligned}
& d G^{+} / d t=+\lambda G^{+}(t), \quad t<t_{D}, \\
& d G^{-} / d t=-\lambda G^{-}(t), \quad t>t_{D} .
\end{aligned}
$$

These equations are very useful to reduce the integrodifferential Eqs. (30) and (31) into the set of differential equations (34)-(36) (with delay); see also Ref. [29].

From Laplace's distribution, Eq. (A1), the mean delay time and its variance are given by

$$
\begin{aligned}
& \langle s\rangle=\mathcal{N}\left(\frac{e^{-\lambda t_{D}}}{\lambda}+2 t_{D}\right), \\
& \sigma^{2} \equiv\left\langle s^{2}\right\rangle-\langle s\rangle^{2}=2\left(\frac{1}{\lambda^{2}}+\mathcal{N} t_{D}^{2}\right)-\mathcal{N}^{2}\left(\frac{e^{-\lambda t_{D}}}{\lambda}+2 t_{D}\right)^{2} .
\end{aligned}
$$

Therefore the variance $\sigma^{2} \equiv \sigma^{2}\left(\lambda, t_{D}\right)$ as function of the two parameters has interesting behaviors to be explored. For example, for fixed $\lambda$ the variance saturates at large $t_{D}$, and for fixed $t_{D}$ the variance goes to zero for large $\lambda$.

Note that for any value of the parameters $\lambda, t_{D}$ the normalization constant $\mathcal{N} \in\left(1, \frac{1}{2}\right)$; it is also simple to see that

$$
\langle s\rangle \rightarrow\left\{\begin{array}{ll}
1 / \lambda, & \left(t_{D} \rightarrow 0, \lambda \neq \infty\right) \\
t_{D}, & \left(t_{D} \gg 1, \lambda \neq 0\right) \\
1 / \lambda, & \left(\lambda \ll 1, t_{D}<\infty\right) \\
t_{D}, & \left(\lambda \rightarrow \infty, t_{D} \neq 0\right)
\end{array} .\right.
$$

The dispersion $\left\langle s^{2}\right\rangle-\langle s\rangle^{2}$ has the asymptotic behavior

$$
\sigma^{2} \rightarrow \begin{cases}1 / \lambda^{2}, & \left(t_{D} \rightarrow 0, \lambda \neq \infty\right) \\ 2 / \lambda^{2}, & \left(t_{D} \rightarrow \infty, \lambda \neq 0\right) \\ 0, & \left(\lambda \rightarrow \infty, t_{D} \neq 0\right)\end{cases}
$$

For completeness we present here the expansion of $G(0)$ and $G\left(t_{D}\right)$ for small $t_{D}$ :

$$
\begin{aligned}
G(0)= & \mathcal{N} \lambda \exp \left(-\lambda t_{D}\right)=\lambda-2 \lambda^{2} t_{D}+3 \lambda^{3} t_{D}^{2} \\
& -\frac{13}{3} \lambda^{4} t_{D}^{3}+O\left(t_{D}^{4}\right), \\
G\left(t_{D}\right)= & \mathcal{N} \lambda=\lambda-\lambda^{2} t_{D}+\frac{3}{2} \lambda^{3} t_{D}^{2}-\frac{13}{6} \lambda^{4} t_{D}^{3}+O\left(t_{D}^{4}\right) .
\end{aligned}
$$


In the exponential case (i.e., when $\left.t_{D}=0\right), G(t)$ fulfills $d G(t) / d t=-\lambda G(t)$, then system Eqs. (3) and (4) is reduced to the Thornley and France's equations:

$$
\begin{aligned}
& \frac{d N}{d t}=N(1-N / K), \\
& \frac{d K}{d t}=\lambda(N-K) .
\end{aligned}
$$

\section{APPENDIX B: ON THE STABILITY EQUATION FOR $\boldsymbol{t}_{\boldsymbol{D}} \neq \mathbf{0}$}

The general condition to study the stability of Eqs. (3) and (6), around the attractor $N=K=\bar{N} \neq 0$, considering a perturbation of the form $n(t) \propto \exp (-c t+i \omega t)$ is given [for any $G(s)$ ] by the equation

$$
\begin{aligned}
& z=1-\int_{0}^{\infty} G(s) e^{z s} d s, \\
& z=c+i \omega .
\end{aligned}
$$

Using the distribution Eq. (9) to make the integral in Eq. (B1) we get

$$
\begin{aligned}
z & =1-\int_{0}^{t_{D}} G(s) e^{z s} d s-\int_{t_{D}}^{\infty} G(s) e^{z s} d s \\
& =1-\mathcal{N} \lambda e^{-\lambda t_{D}}\left(\frac{e^{(z+\lambda) t_{D}}-1}{z+\lambda}-\frac{e^{(z+\lambda) t_{D}}}{z-\lambda}\right) \\
& =1-\left[\frac{G\left(t_{D}\right) e^{z t_{D}}-G(0)}{z+\lambda}-\frac{G\left(t_{D}\right) e^{z t_{D}}}{z-\lambda}\right] .
\end{aligned}
$$

This expression can be put in the following alternative form, which is useful for studying different limits:

$$
\begin{aligned}
(z-\lambda)(z-1) & =\left[G(0)-G\left(t_{D}\right) e^{z t_{D}}\right] \frac{(z-\lambda)}{(z+\lambda)}+G\left(t_{D}\right) e^{z t_{D}} \\
& =G(0) \frac{(z-\lambda)}{(z+\lambda)}+G\left(t_{D}\right) e^{z t_{D}} \frac{2 \lambda}{(z+\lambda)} .
\end{aligned}
$$

\section{APPENDIX C: ON THE EVALUATION OF $N(\infty)$ FOR THE CASE $t_{D} \neq 0$}

To interpret the value of the attractor $N(\infty)=\bar{N}$ we integrate formally Eq. (36), and we get

$$
\begin{aligned}
\int_{0}^{t} \dot{V}(s) d s= & G(0) \int_{0}^{t} \dot{N}(s) d s-2 \lambda G\left(t_{D}\right) \int_{0}^{t} N\left(s-t_{D}\right) d s \\
& +\lambda G(0) \int_{0}^{t} N(s) d s+\lambda^{2} \int_{0}^{t} K(s) d s \\
= & G(0)[N(t)-N(0)]-2 \lambda G\left(t_{D}\right) \\
& \times\left[\int_{0}^{t_{D}} N\left(s-t_{D}\right) d s+\int_{t_{D}}^{t} N\left(s-t_{D}\right) d s\right] \\
& +\lambda G(0) \int_{0}^{t} N(s) d s+\lambda^{2} \int_{0}^{t} K(s) d s ; \quad(\mathrm{C} 1)
\end{aligned}
$$

taking $t \rightarrow \infty$ and using $V(\infty)=0$ we obtain

$$
\begin{aligned}
-V(0)= & G(0)[N(\infty)-N(0)]-2 \lambda G\left(t_{D}\right) \int_{-t_{D}}^{0} N(x) d x \\
& +\left[\lambda G(0)-2 \lambda G\left(t_{D}\right)\right] \int_{0}^{\infty} N(x) d x \\
& +\lambda^{2} \int_{0}^{\infty} K(s) d s .
\end{aligned}
$$

Noting that $\lambda G(0)-2 \lambda G\left(t_{D}\right)=-\lambda^{2}$, using $V(0)=G(0)$ $[N(0)-\varphi]$, and assuming a constant prefunction $[\varphi=K(0)]$ in Eq. (C2) we arrive at

$$
\begin{aligned}
& G(0)[\bar{N}-K(0)] \\
& \quad=\lambda^{2} \int_{0}^{\infty}[N(s)-K(s)] d s+2 \lambda G\left(t_{D}\right) t_{D} K(0) .
\end{aligned}
$$

This is an exact result which gives an Areas's rule for $\bar{N}$ in the case $t_{D} \neq 0$; this result reduces to Eq. (20) in the limit $t_{D} \rightarrow 0$. An equivalent formula can also be obtained in the general case when the prefunction $\varphi(s)$ is not a constant.

\section{APPENDIX D: STABILITY CONDITION WHEN $\lambda \rightarrow \infty$ WITH $t_{D} \neq 0$}

In this particular situation we get $G(s) \rightarrow \delta\left(s-t_{D}\right)$; therefore our generalized delay-coupled Logistic-capacity model looks like

$$
\begin{aligned}
\dot{N} & =N(1-N / K), \\
K(t) & =N\left(t-t_{D}\right) .
\end{aligned}
$$

Putting $N(t) \sim \bar{N}(1+n(t))$ in Eqs. (D1) and (D2) the linear stability condition follows from the analysis of

$$
\dot{n}(t)=-n(t)+n\left(t-t_{D}\right) .
$$

Using $n(t) \propto e^{(-c+i \omega) t}$ gives the equation $(-c+i \omega)=-1+$ $e^{-(-c+i \omega) t_{D}}$, which is in agreement with Eq. (29).

It is well known [30,31] that a necessary and sufficient condition for the stability of any solution of

$$
\dot{n}(t)=-a n(t)+b n\left(t-t_{D}\right)
$$

is

$$
t_{D}<\frac{\cos ^{-1}(a / b)}{\sqrt{b^{2}-a^{2}}} .
$$

This inequality defines the domain of stability, whose boundaries, in the plane $(a, b)$, are given by the line $a=b$, and the curve defined parametrically as $a=-\omega / \tan \left(\omega t_{D}\right)$ and $b=-\omega / \sin \left(\omega t_{D}\right)$, where for a given $t_{D}$ it follows that $\omega t_{D} \in(0, \pi)$. These two boundaries intersect at the point $\left(-1 / t_{D},-1 / t_{D}\right)$. From these results it follows that in the limit $\lambda \rightarrow \infty$ (with $t_{D}<1$ ) our system is at the boundary of stability.

Note that the parameters $a, b$ in Eq. (D4) do not appear in our case, but it could be of interest to define a related model where $K(t) \propto N\left(t-t_{D}\right)$, in which case new constants will appear in the linear stability analysis, changing the condition Eq. (D3) into the form (D4). 
[1] T. R. Malthus, An Essay on the Principle of Population (J. Johnson, London, St. Paul's Church Yard, 1798).

[2] P. F. Verhulst, Corres. Math. Phys. 10, 113 (1838).

[3] V. Volterra, Memoria della R. Accad. Nazl. Lincei, Ser. VI 2, 31 (1926) [Translated in R. Chapman, Animal Ecology (McGrawHill, New York, 1931), pp. 409-447].

[4] N. S. Goel and N. Richter-Dyn, Stochastic Models in Biology (Academic Press, New York, 1974).

[5] J. H. Matis, T. T. Kiffe, E. Renshaw, and J. Hassan, Ecol. Model. 161, 239 (2003).

[6] H. M. Hastings, D. J. Rumignani, A. G. Vassolatti, M. T. Weiss, and Y. R. Wu, Ecol. Model. 92, 209 (1996).

[7] S. Invernizzi and K. Terpin, Ecol. Model. 94, 231 (1997).

[8] C. P. D. Birch, Ann. Bot. 83, 713 (1999).

[9] H. Fujikawa, A. Kai, and S. Morozumi, J. Food Hyg. Soc. Jnp. 44, 155 (2003).

[10] J. H. M. Thornley and J. France, Ecol. Model. 184, 257 (2005).

[11] H. M. Safuan, I. N. Towers, Z. Jovanoski, and H. S. Sidhu, ANZIAM J. 53, (EMAC2011), C172 (2012).

[12] M. O. Cáceres, J. Stat. Phys. 132, 487 (2008).

[13] N. G. van Kampen, Stochastic Processes in Physics and Chemistry, 2nd ed. (North Holland, Amsterdam, 1992).

[14] M. O. Cáceres, Elementos de estadistica de no equilibrio y sus aplicaciones al transporte en medios desordenados (Reverté S. A., Barcelona, 2003).

[15] R. L. Stratonovich, Topics in the Theory of Random Noise (Gordon and Breach, New York, 1963), Vols. 1-2.

[16] J. R. Beddington and R. M. May, Math. Biosci. 27, 109 (1975).
[17] J. D. Murray, Mathematical Biology, 3rd ed. (Springer, Berlin, 2007), Vol. 1.

[18] E. Ben-Jacob, I. Cohen, and H. Levine, Adv. Phys. 49, 395 (2000).

[19] R. A. Fisher, Ann. Eugen. Lond. 7, 355 (1937).

[20] M. A. Fuentes, M. N. Kuperman, and V. M. Kenkre, Phys. Rev. Lett. 91, 158104 (2003).

[21] E. Hernandez-Garcia and C. Lopez, Phys. Rev. E 70, 016216 (2004).

[22] M. G. Clerc, D. Escaff, and V. M. Kenkre, Phys. Rev. E 72, 056217 (2005); 82, 036210 (2010).

[23] M. A. Fuentes and M. O. Cáceres, Cent. Eur. J. Phys. 11, 1623 (2013).

[24] S. Goncalves, G. Abranson, and M. F. C. Gomes, Eur. Phys. J. B 81, 363 (2011).

[25] I. Ikeda and T. Yokoi, Ecol. Model. 10, 141 (1980).

[26] C. V. Trappey and H. Y. Wu, Adva. Eng. Inf. 22, 421 (2008).

[27] S. P. Rogovchenko and Y. V. Rogovchenko, Chaos, Solitons Fractals 39, 1169 (2009).

[28] H. M. Safuan, I. N. Towers, Z. Jovanoski, and H. S. Sidhu, in Proceedings of the 19th International Congress on Modelling and Simulation, edited by F. Chan, D. Marinova, and R. S. Anderssen (2011), pp. 733-739.

[29] M. O. Cáceres, J. Stat. Phys. 156, 94 (2014).

[30] R. D. Drivers, Ordinary and Delay Differential Equations (Springer, Berlin, 1993).

[31] A. A. Budini and M. O. Cáceres, Phys. Rev. E 70, 046104 (2004). 